\title{
THE SILENT DISEASE
}

Kidney cancer is being detected earlier thanks to modern diagnostic techniques, meaning more patients receive treatment. By Graham Shaw; illustration by Lucy Reading-Ikkanda

\section{KIDNEY CANCER, BY THE NUMBERS}

Kidney cancers are a range of diseases that affect the body's ability to filter waste products in the blood. They often develop without symptoms, which means they are normally picked up in tests for other reasons. The disease is one of the ten most common forms of cancer in developed countries, for reasons that are not well understood.

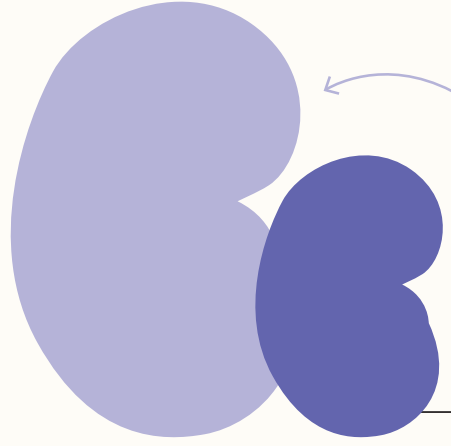

In 2012, worldwide ${ }^{1}$

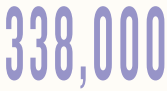

new cases of kidney cancer

143.000

deaths from kidney cancer
Men are twice as likely as

women to develop kidney cancer

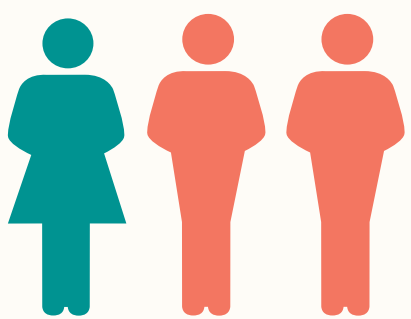

\section{COMMON FORMS}

Renal cell carcinoma (RCC) is the most common form of kidney cancer and affects tubules inside the kidney that transfer waste products from the blood to the urine through the glomerulus. RCC has multiple subtypes, of which clear-cell carcinoma is the most common. The remaining $10 \%$ of cancers are transitional cell carcinomas, which form in cells lining the renal pelvis and ureter ${ }^{2}$.

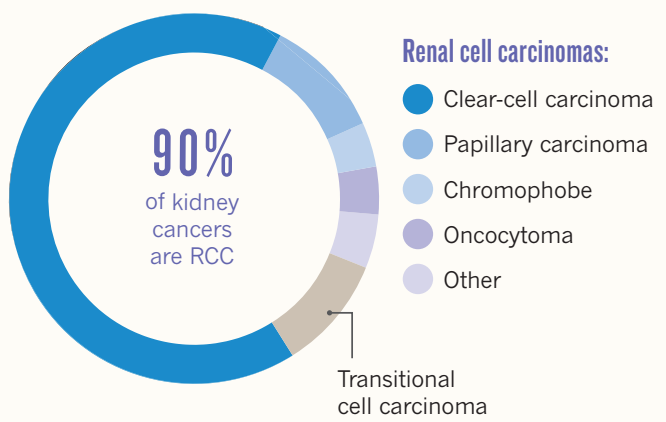

\section{GLOBAL KIDNEY CANCER RATES}

Kidney cancer is more common in some parts of the world than in others ${ }^{3}$. People from developed countries are more than four times as likely to develop RCC than people from developing nations. Generally, there is less geographical variation for transitional cell cancers.

Kidney cancer rates per 100,000

South Africa

Malaysia

Egypt

China

Iceland

Italy

Norway

Canada

Russia

Australia

Uruguay

France

Israel

Germany

Slovenia

Latvia

United States

Slovakia

Lithuania

Czech Republic
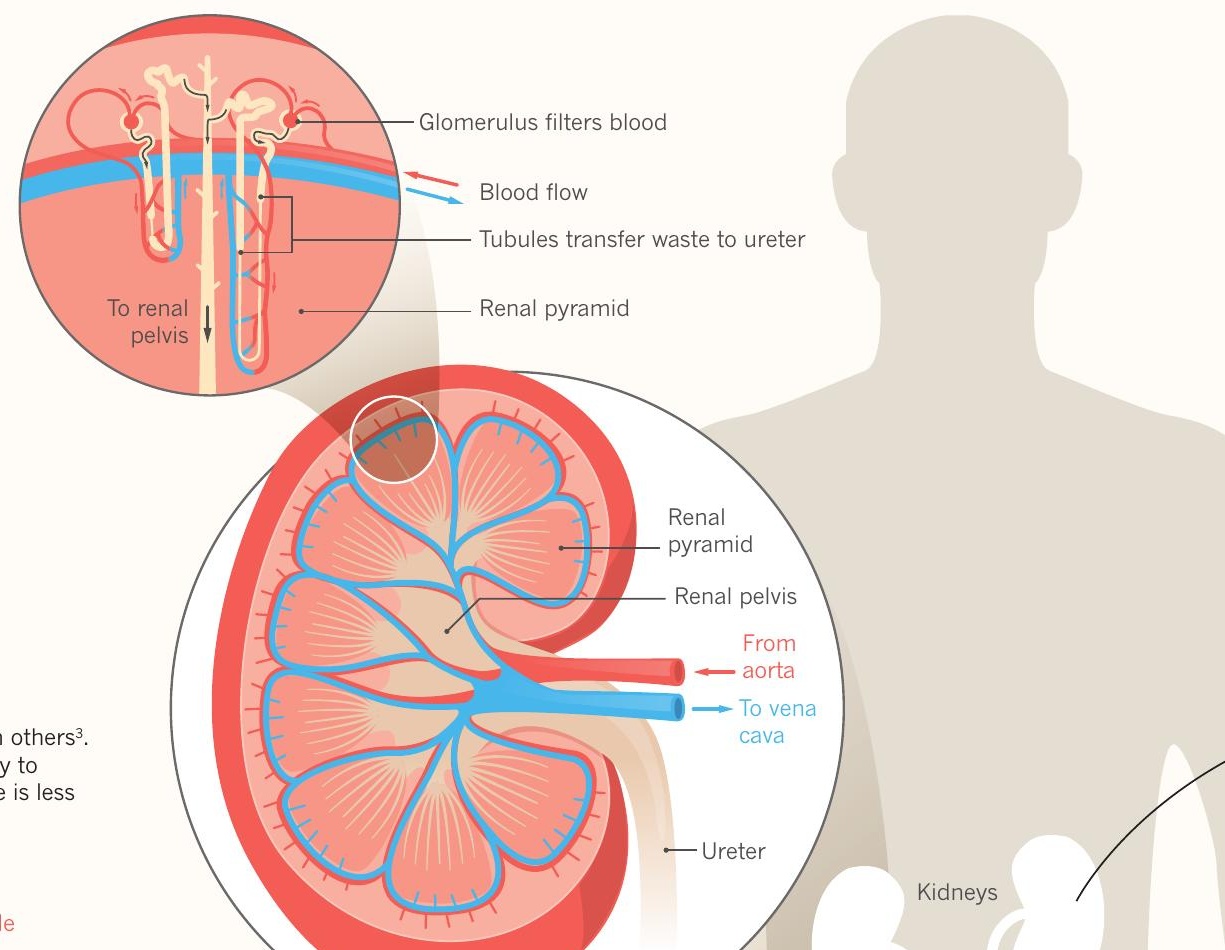


\section{TRIGGER WARNING}

Smoking and obesity are the biggest risk factors for kidney cancer.

\section{Smoking}

The falling popularity of smoking in developed countries could help to lower kidney-cancer incidence in these regions ${ }^{4}$.

\section{Obesity}

Every five-point increase in body mass index (BMI) increases risk by $24 \%$ for men and $34 \%$ for women. The rising level of obesity worldwide is likely to have contributed to increases in kidney cancer ${ }^{4}$.

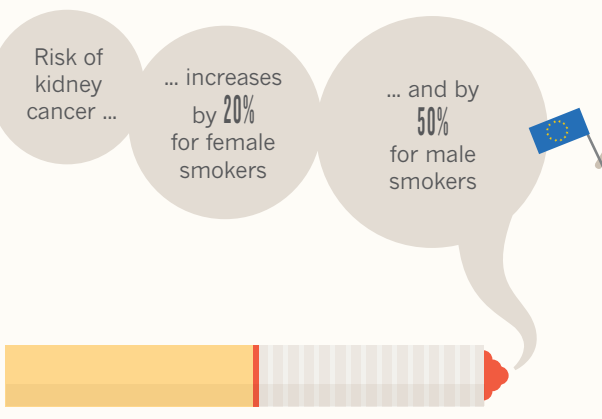

Percentage of people with renal-cell cancers who are overweight ${ }^{4}$

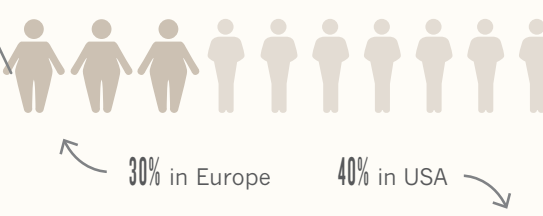

\section{Hypertension}

Risk of kidney cancer increases with rising blood pressure. Although obesity can increase blood pressure, the two risk factors are independent and the chance of developing the disease is higher among individuals with both conditions than for those who have one ${ }^{4}$

\section{DEVELOPING STORY}

Loss-of-function mutations in the gene $\mathrm{VHL}$ are responsible for about $60 \%$ of clear-cell carcinomas ${ }^{2}$. Around $2-3 \%$ of renal cell carcinomas run in families ${ }^{5}$. People with an inherited form of kidney cancer caused by von Hippel-Lindau syndrome $(\mathrm{VHL})$ are born with a defect to an allele of VHL, and go on to acquire mutations that silence the gene. Most people with kidney cancer acquire VHL mutations during their lives as a result of environmental factors. A lack of symptoms can mean that some cancers can spread to other parts of the body before they are caught.

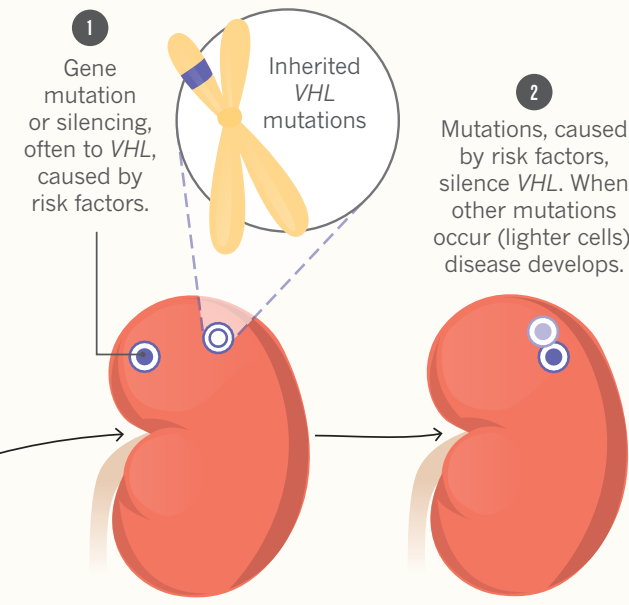

\section{Mutations lead to blood-vessel formation}

The VHL protein is a tumour suppressor, but this protein is missing in mutated cells. When this happens, the transcription factor HIF- $\alpha$ accumulates in cancer cells, leading to the overexpression of proteins (such as VEGF and TGF- $\alpha$ ) that are normally only produced in low-oxygen environments.

The protein TGF- $\alpha$ acts on cancer cells to promote proliferation and survival.

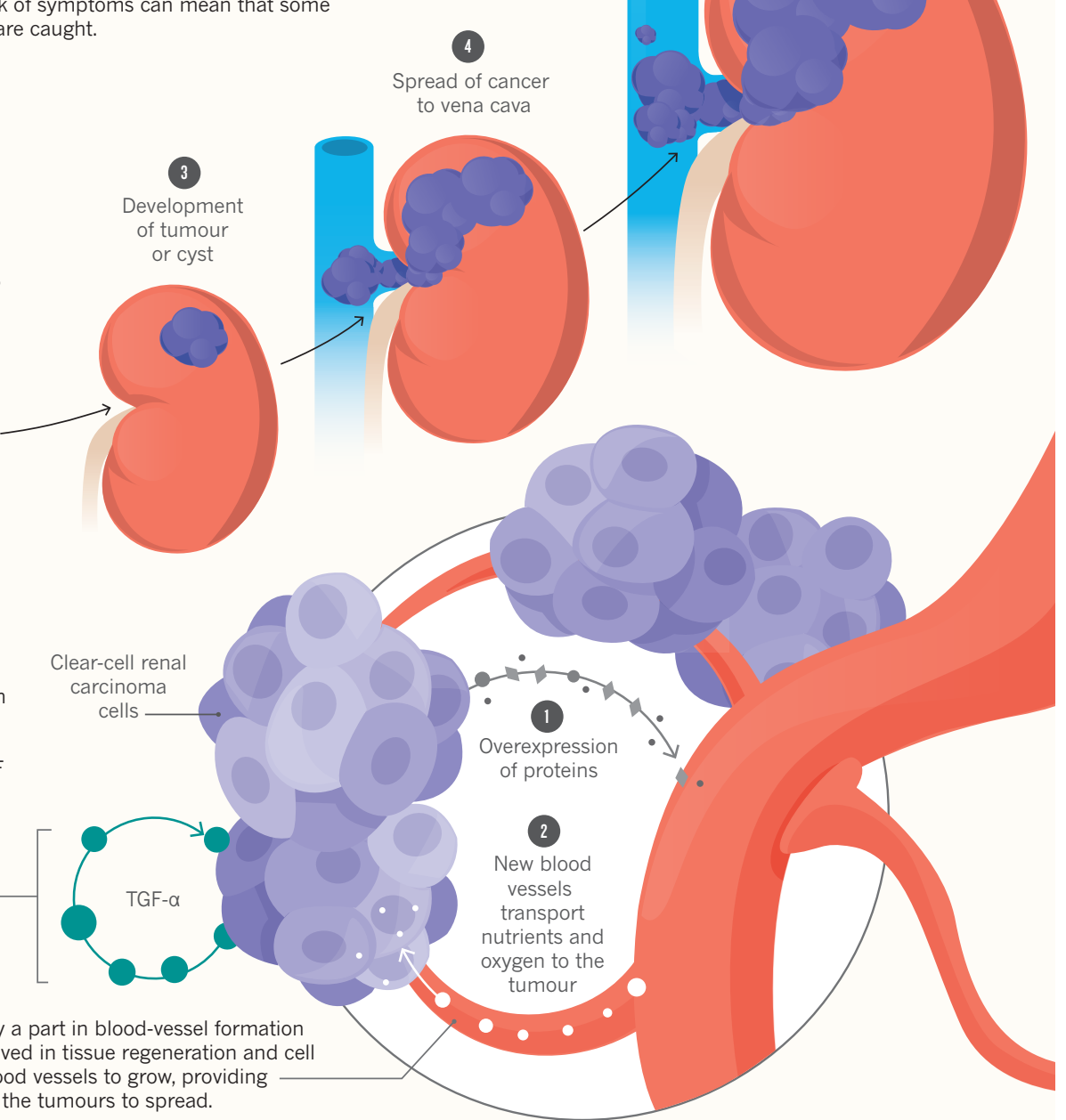

PDGFB and VEGF (which play a part in blood-vessel formation and growth) and TGF- $\beta$ (involved in tissue regeneration and cell differentiation) cause new blood vessels to grow, provic
nutrients and oxygen to help the tumours to spread.

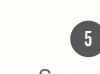

Spread of cancer to organs such as the lymph nodes

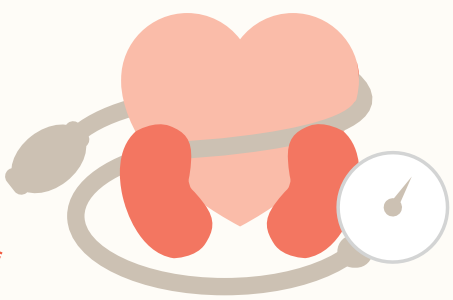

